\title{
The First Synthesis of Biquinoliziniun Salts: A Novel Example of a Chiral Azonia Dication
}

\author{
Domingo García-Cuadrado, ${ }^{\dagger}$ Ana M. Cuadro, ${ }^{*}{ }^{\dagger}$ Julio Alvarez-Builla, ${ }^{\dagger}$ Unai Sancho, ${ }^{*}$ \\ Obis Castaño, ${ }^{\star}$ and Juan J. Vaquero ${ }^{* \dagger}$ \\ ${ }^{\dagger}$ Departamento de Química Orgánica and Química Física, Universidad de Alcalá \\ 28871 Alcalá de Henares. Madrid (Spain)
}

\begin{abstract}
Supporting Information
Experimental procedures, characterization data and computacional data for biquinolizinium dibromides 1-4
\end{abstract}

(17 pages )

\section{Experimental section}

General. Melting points were uncorrected. Infrared spectra were recorded on $\mathrm{KBr}$ or $\mathrm{NaCl}$ pellets and spectral bands were reported in $\mathrm{cm}^{-1}$. ${ }^{1} \mathrm{H}$ NMR spectra were recorded at 200, 300 and $500 \mathrm{MHz}$ and ${ }^{13} \mathrm{C}$ NMR at 75 and $125 \mathrm{MHz}$. Assignments for 1-3 were made by gHMQC and TOCSY1D experiments. Chemical shifts were reported as $\delta$ values (ppm). Lowresolution mass spectra (MS) were obtained as ESI (Na). CuI, $\mathrm{PdCl}_{2}\left(\mathrm{PPh}_{3}\right)_{2}$, were purchased from Aldrich. Bromoquinolizinium bromides 5-8 were obtained by previously described methods. ${ }^{11} \mathrm{DMF}, \mathrm{DMAC}$ and $\mathrm{Et}_{3} \mathrm{~N}$ were distilled over activated molecular sieves.

\section{Synthesis of 1-4. General procedure}

To a solution of the corresponding bromoquinoliziniun bromides $\mathbf{5 - 8}(100 \mathrm{mg}, 0.346 \mathrm{mmol})$ and $10 \% \mathrm{~mol} \mathrm{CuI} \mathrm{(6.6} \mathrm{mg,} 0.0346 \mathrm{mmol})$ in dry DMF (3 mL) under argon, hexamethyldistannane (113.3 mg, $0.346 \mathrm{mmol})$ in dry DMF $(2 \mathrm{~mL})$ and $\mathrm{Pd}\left(\mathrm{PPh}_{3}\right)_{4}(20.0 \mathrm{mg}, 0.0173 \mathrm{mmol})$ were added and the reaction mixture was stirred at room temperature for $22 \mathrm{~h}$. After, the solution was concentrated under reduced pressure and the residue treated with $3 \mathrm{~mL}$ of EtOH (1 and $\mathbf{3}$ ) or THF (2). The solid was filtered and washed with ethanol (1 and 3) or AcOEt (2). Purification of the crude product by column chromatography on silica gel (reverse phase) using water as eluent, yielded the corresponding biquinolizinium dibromides 1-3.

(11) (a) Sanders, G. M.; van Dijk, M.; van der Plas, H. C. Heterocycles 1981, 15, 213 and references therein. (b). Glover, E. E; Jones, G. J. Chem. Soc. 1958, 3021 


\section{1,1'-Biquinolizinium Dibromide (1)}

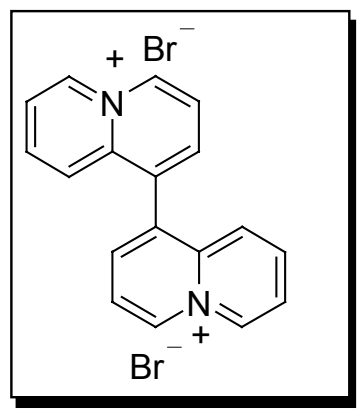

$19.4 \mathrm{mg}$ (26\%); white solid; mp.: $>350{ }^{\circ} \mathrm{C}$. IR $(\mathrm{KBr}): v_{\max }\left(\mathrm{cm}^{-1}\right)$ 3001, 1639, 1421, 1402, 1363, 1155, 818, 617. ${ }^{1} \mathrm{H}$ NMR (300 MHz, $\left.\mathrm{D}_{2} \mathrm{O}\right) \delta(\mathrm{ppm}) 9.13(\mathrm{~d}, 2 \mathrm{H}, J=7.0 \mathrm{~Hz}), 9.10(\mathrm{~d}, 2 \mathrm{H}, J=6.6 \mathrm{~Hz}), 8.21$ (d, 2H, $J=7.5 \mathrm{~Hz}), 8.05-7.96(\mathrm{~m}, 4 \mathrm{H}), 7.88(\mathrm{t}, 2 \mathrm{H}, J=6.5 \mathrm{~Hz}), 7.68$ $(\mathrm{d}, 2 \mathrm{H}, J=8.8 \mathrm{~Hz}) \cdot{ }^{13} \mathrm{C}$ NMR $\left(75 \mathrm{MHz}, \mathrm{D}_{2} \mathrm{O}\right) \delta(\mathrm{ppm}) \quad 142.4,139.4$, $138.6,138.1,137.7,131.7,124.7,124.6,123.5 . \mathrm{MS}\left(\mathrm{ES}^{+}\right) \mathrm{m} / \mathrm{z}$ (relative intensity) $337\left(\mathrm{M}^{2+}+\mathrm{Br}^{-}, 29\right), 129\left(\mathrm{M}^{2+} / 2,100\right)$. Anal. Calcd for $\mathrm{C}_{18} \mathrm{H}_{14} \mathrm{Br}_{2} \mathrm{~N}_{2} \cdot \mathrm{H}_{2} \mathrm{O}$ (436.14g/mol): C (49.57), H (3.70), N (6.42). Found C (49.83), H (3.41), $\mathrm{N}(6.68)$.

\section{2,2'-Biquinolizinium Dibromide (2)}

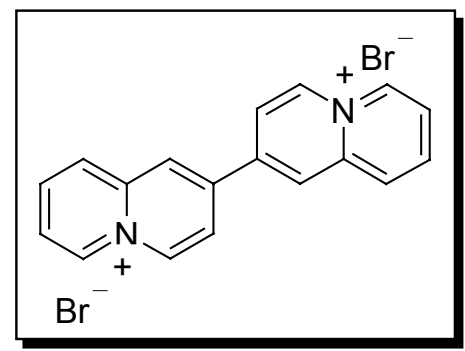

$40.4 \mathrm{mg}$ (56\%); white solid; mp.:>350 ${ }^{\circ} \mathrm{C}$. IR (KBr): $\mathrm{v}_{\max }$ $\left(\mathrm{cm}^{-1}\right) 3102,3008,1647,1390,1140,824,768 .{ }^{1} \mathrm{H}$ NMR (500 MHz, DMSO- $\left.d_{6}\right) \delta(\mathrm{ppm}) 9.60(\mathrm{~d}, 2 \mathrm{H}, J=7.0 \mathrm{~Hz}), 9.46(\mathrm{~d}$, $2 \mathrm{H}, J=6.0 \mathrm{~Hz}), 9.42(\mathrm{~s}, 2 \mathrm{H}), 8.76(\mathrm{dd}, 2 \mathrm{H}, J=7.0,2.0 \mathrm{~Hz})$, $8.67(\mathrm{~d}, 2 \mathrm{H}, J=7.5 \mathrm{~Hz}), 8.54(\mathrm{td}, 2 \mathrm{H}, J=7.6,1.0 \mathrm{~Hz}), 8.24$ $(\operatorname{td}, 2 \mathrm{H}, J=6.5,2.0 \mathrm{~Hz}) \cdot{ }^{13} \mathrm{C}$ NMR $\left(125 \mathrm{MHz}, \mathrm{DMSO}-d_{6}\right) \delta$ (ppm) 142.2, 140.2, 138.0, 137.3, 137.0, 127.6, 125.4, 124.6, 120.6. $\mathrm{MS}\left(\mathrm{ES}^{+}\right) \mathrm{m} / \mathrm{z}$ (relative intensity) $337\left(\mathrm{M}^{2+}+\mathrm{Br}^{-}, 56\right), 258$ $\left(\mathrm{M}^{2+}, 28\right), 129\left(\mathrm{M}^{2+} / 2,100\right)$. Anal. Calcd for $\mathrm{C}_{18} \mathrm{H}_{14} \mathrm{Br}_{2} \mathrm{~N}_{2}$ (418.13g/mol): C (51.71), H (3.37), N (6.70). Found C (51.60), $\mathrm{H}(3.16), \mathrm{N}(6.79)$.

\section{3,3'-Biquinolizinium Dibromide (3)}

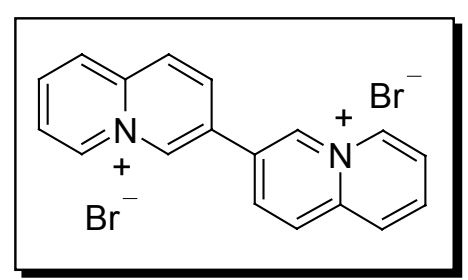

$40.4 \mathrm{mg}$ (56\%); white solid; mp.:>350 ${ }^{\circ} \mathrm{C}$. IR (KBr): $\mathrm{v}_{\max }$ $\left(\mathrm{cm}^{-1}\right) 3015,1649,1634,1432,1171,839,486 .{ }^{1} \mathrm{H}$ NMR (300 $\left.\mathrm{MHz}, \mathrm{D}_{2} \mathrm{O}\right) \delta(\mathrm{ppm}) \delta 9.46(\mathrm{~s}, 2 \mathrm{H}), 9.05(\mathrm{~d}, 2 \mathrm{H}, J=6.6 \mathrm{~Hz})$, 8.51 (d, 2H, $J=9.3 \mathrm{~Hz}), 8.43$ (d, 2H, $J=9.2 \mathrm{~Hz}), 8.35$ (d, 2H, $J=9.0 \mathrm{~Hz}), 8.24(\mathrm{t}, 2 \mathrm{H}, J=8.1 \mathrm{~Hz}), 7.92(\mathrm{t}, 2 \mathrm{H}, J=6.7 \mathrm{~Hz})$. ${ }^{13} \mathrm{C}$ NMR $\left(75 \mathrm{MHz}, \mathrm{D}_{2} \mathrm{O}\right) \delta(\mathrm{ppm}) 144.4,139.8,138.5,136.7$, 132.0, 129.5, 128.8, 126.3. MS (ES $\left.{ }^{+}\right) \mathrm{m} / z$ (relative intensity) $337\left(\mathrm{M}^{2+}+\mathrm{Br}^{-}, 41\right), 129\left(\mathrm{M}^{2+} / 2,100\right)$. Anal. Calcd for $\mathrm{C}_{18} \mathrm{H}_{14} \mathrm{Br}_{2} \mathrm{~N}_{2} . \mathrm{H}_{2} \mathrm{O}$ (436.14g/mol): C (49.57), H (3.70), N (6.42). Found C (49.84), H (3.52), N (6.63). 


\section{Computacional Data}

All the calculations were carried out using the Gaussian 98 suite of quantum chemical programs. ${ }^{12}$ The relaxed scan calculations were carried out for the rotation angles, in the four molecules studied, around the 360 degrees, within a step of 10 degrees at the Hartree-Fock level of theory using a $6-31 \mathrm{G}(\mathrm{d})$ basis set. The stationary points identified result being minima after its geometry optimizations and characterization by frequency calculations. In our search, we also found the transition states between all the identified minima, being the calculations also at the same level of theory.

Relative energies were calculated at $0 \mathrm{~K}$ and recalculated at $298 \mathrm{~K}$ including the Zero Point Energies (ZPE), the thermal corrections (TCE) and the corresponding scaling factor equal to 0.9135 .

(13) Gaussian 98, Revision A.11, Frisch, M. J.; Trucks, G. W.; Schlegel, H. B.; Scuseria, G. E.; Robb, M. A.; Cheeseman, J. R.; Zakrzewski, V. G.; Montgomery Jr., J. A.; Stratmann, R. E.; Burant, J. C.; Dapprich, S.; Millam, J. M.; Daniels, A. D.; Kudin, K. N.; Strain, M. C.; Farkas, O.; Tomasi, J.; Barone, V.; Cossi, M.; Cammi, R.; Mennucci, B.; Pomelli, C.; Adamo, C.; Clifford, S.; Ochterski, J.; Petersson, G. A.; Ayala, P. Y.; Cui, Q.; Morokuma, K.; Salvador, P.; Dannenberg, J. J.; Malick, D. K.; Rabuck, A. D.; Raghavachari, K.; Foresman, J. B.; Cioslowski, J.; Ortiz, J. V.; Baboul, A. G.; Stefanov, B. B.; Liu, G.; Liashenko, A.; Piskorz, P.; Komaromi, I.; Gomperts, R.; Martin, R. L.; Fox, D. J.; Keith, T.; Al-Laham, M. A.; Peng, C. Y.; Nanayakkara, A.; Challacombe, M.; Gill, P. M. W.; Johnson, B.; Chen, W.; Wong, M. W.; Andres, J. L.; Gonzalez, C.; Head-Gordon, M.; Replogle, E. S.; Pople, J. A.; Gaussian, Inc.: Pittsburgh PA, 2001. 


\section{$\underline{\text { 1,1'-Biquinolizinium GS }}$}

$\begin{array}{rrrr}6 & -3.827958 & -1.143697 & -1.602055 \\ 6 & -4.002638 & -0.130172 & -0.734998 \\ 6 & -2.551058 & -1.738611 & -1.710885 \\ 1 & -4.655581 & -1.484598 & -2.192829 \\ 7 & -2.966281 & 0.338217 & 0.045140 \\ 6 & -1.527571 & -1.279984 & -0.942253 \\ 1 & -4.941256 & 0.366176 & -0.598213 \\ 1 & -2.394076 & -2.549953 & -2.396382 \\ 6 & -3.231601 & 1.381606 & 0.913775 \\ 6 & -1.723026 & -0.216413 & -0.035338 \\ 1 & -0.556249 & -1.725033 & -1.006791 \\ 6 & -2.275945 & 1.883453 & 1.707529 \\ 6 & -0.686375 & 0.306391 & 0.805765 \\ 1 & -4.238308 & 1.745539 & 0.903000 \\ 6 & -0.971669 & 1.328663 & 1.653609 \\ 6 & 0.686375 & -0.306391 & 0.805765 \\ 1 & -2.506125 & 2.689978 & 2.375775 \\ 6 & 1.723026 & 0.216413 & -0.035338 \\ 6 & 0.971669 & -1.328663 & 1.653609 \\ 1 & -0.208366 & 1.724122 & 2.297882 \\ 7 & 2.966281 & -0.338217 & 0.045140 \\ 6 & 2.275945 & -1.883453 & 1.707529 \\ 6 & 1.527571 & 1.279984 & -0.942253 \\ 1 & 0.208366 & -1.724122 & 2.297883 \\ 6 & 3.231601 & -1.381606 & 0.913775 \\ 6 & 2.551058 & 1.738611 & -1.710885 \\ 6 & 4.002638 & 0.130172 & -0.734998 \\ 1 & 2.506125 & -2.689978 & 2.375775 \\ 1 & 0.556249 & 1.725033 & -1.006791 \\ 6 & 3.827958 & 1.143697 & -1.602055 \\ 1 & 4.238308 & -1.745539 & 0.903000 \\ 1 & 2.394076 & 2.549953 & -2.396382 \\ 1 & 4.941256 & -0.366176 & -0.598213 \\ 1 & 4.655581 & 1.484598 & -2.192829\end{array}$

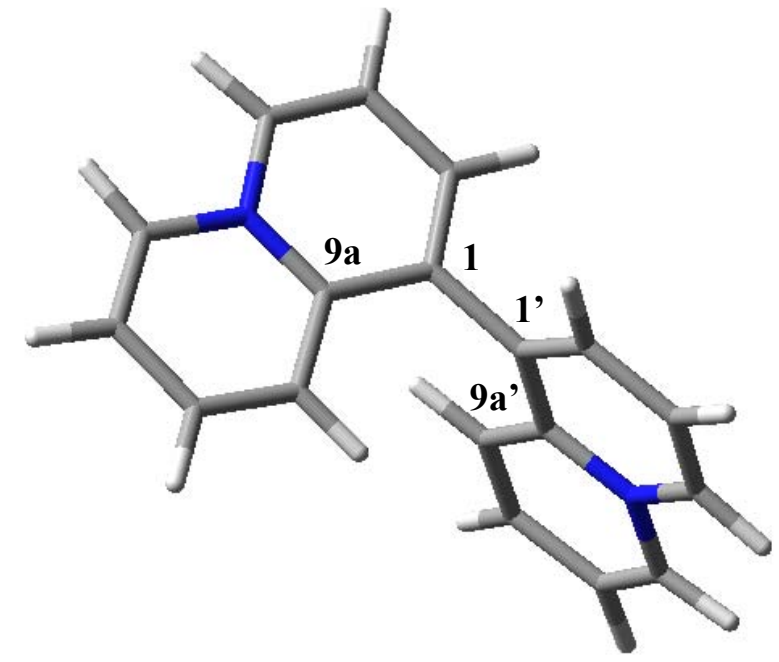

$\mathrm{E}_{\mathrm{HF} / 6-31 \mathrm{G}(\mathrm{d})}(\mathrm{a} . \mathrm{u})=-798.1876659$ ZPE (a.u.) $=0.298376$

Number of Imaginary Frequencies $=0$ 


\section{$\underline{\text { 1,1'-Biquinolizinium anti-TS }}$}

$\begin{array}{rrr}4.651019 & -0.929736 & 0.157403 \\ 4.305780 & 0.362739 & 0.291174 \\ 3.652462 & -1.837360 & -0.242828 \\ 5.671639 & -1.230151 & 0.291044 \\ 3.007423 & 0.792470 & 0.118700 \\ 2.358900 & -1.423184 & -0.344784 \\ 5.016136 & 1.135490 & 0.500876 \\ 3.910588 & -2.850899 & -0.486044 \\ 2.819955 & 2.160021 & 0.023782 \\ 1.977989 & -0.090559 & -0.068749 \\ 1.631785 & -2.106167 & -0.719759 \\ 1.628166 & 2.642943 & -0.331694 \\ 0.613449 & 0.435172 & -0.097535 \\ 3.687400 & 2.764162 & 0.189600 \\ 0.520091 & 1.762682 & -0.400529 \\ -0.607659 & -0.432385 & 0.116525 \\ 1.499054 & 3.693944 & -0.500119 \\ -1.972198 & 0.093348 & 0.087742 \\ -0.514301 & -1.759895 & 0.419517 \\ -0.411203 & 2.199936 & -0.676622 \\ -3.001634 & -0.789679 & -0.099707 \\ -1.622377 & -2.640154 & 0.350681 \\ -2.353107 & 1.425973 & 0.363779 \\ 0.416992 & -2.197151 & 0.695609 \\ -2.814167 & -2.157230 & -0.004792 \\ -3.646668 & 1.840151 & 0.261827 \\ -4.299991 & -0.359946 & -0.272179 \\ -1.493266 & -3.691156 & 0.519104 \\ -1.625990 & 2.108954 & 0.738754 \\ -4.645228 & 0.932529 & -0.138405 \\ -3.681613 & -2.761370 & -0.170610 \\ -3.904793 & 2.853690 & 0.505044 \\ -5.010348 & -1.132696 & -0.481881 \\ -5.665847 & 1.232945 & -0.272044\end{array}$

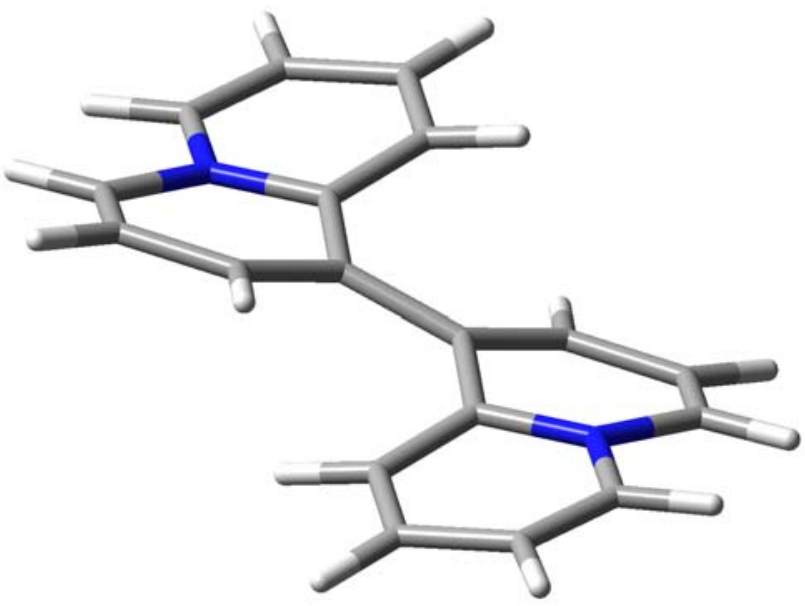

$\mathrm{E}_{\mathrm{HF} / 6-31 \mathrm{G}(\mathrm{d})}(\mathrm{a} . \mathrm{u})=-798.1392413$

$\mathrm{ZPE}$ (a.u.) $=0.298976$

Number of Imaginary Frequencies $=1$ $v\left(\mathrm{~cm}^{-1}\right)=-43.1458$ 


\section{$\underline{\text { 2,2'-Biquinolizinium GS1 }}$}

$\begin{array}{rrrr}6 & 1.492980 & 0.579325 & -0.360070 \\ 6 & 2.911461 & 0.564864 & -0.244735 \\ 6 & 0.742580 & -0.467630 & 0.060847 \\ 1 & 1.049691 & 1.443032 & -0.816650 \\ 7 & 3.510922 & -0.524404 & 0.304094 \\ 6 & 1.414711 & -1.595892 & 0.626426 \\ 6 & 3.712802 & 1.635699 & -0.683345 \\ 6 & -0.742580 & -0.467631 & -0.060848 \\ 6 & 2.749557 & -1.595461 & 0.737143 \\ 6 & 5.065933 & 1.578805 & -0.553909 \\ 6 & -1.414711 & -1.595892 & -0.626426 \\ 6 & -1.492980 & 0.579325 & 0.360070 \\ 6 & 4.879566 & -0.581958 & 0.435759 \\ 1 & 0.867539 & -2.444300 & 0.989391 \\ 1 & 3.228333 & 2.487967 & -1.118505 \\ 6 & 5.662054 & 0.434717 & 0.023996 \\ 6 & -2.911461 & 0.564864 & 0.244735 \\ 6 & -2.749958 & -1.595461 & -0.737143 \\ 1 & 3.299248 & -2.409041 & 1.164481 \\ 1 & 5.680500 & 2.393672 & -0.886975 \\ 1 & -0.867539 & -2.444300 & -0.989391 \\ 1 & -1.049691 & 1.443032 & 0.816649 \\ 1 & 5.265472 & -1.476618 & 0.879702 \\ 7 & -3.510921 & -0.524404 & -0.304094 \\ 6 & -3.712802 & 1.635699 & 0.683345 \\ 1 & 6.725851 & 0.361303 & 0.139232 \\ 1 & -3.299248 & -2.409041 & -1.164480 \\ 6 & -4.879566 & -0.581958 & -0.435759 \\ 6 & -5.065933 & 1.578805 & 0.553909 \\ 1 & -3.228333 & 2.487967 & 1.118505 \\ 6 & -5.662054 & 0.434717 & -0.023996 \\ 1 & -5.265472 & -1.476618 & -0.879702 \\ 1 & -5.680500 & 2.393672 & 0.886975 \\ 1 & -6.725851 & 0.361303 & -0.139232\end{array}$

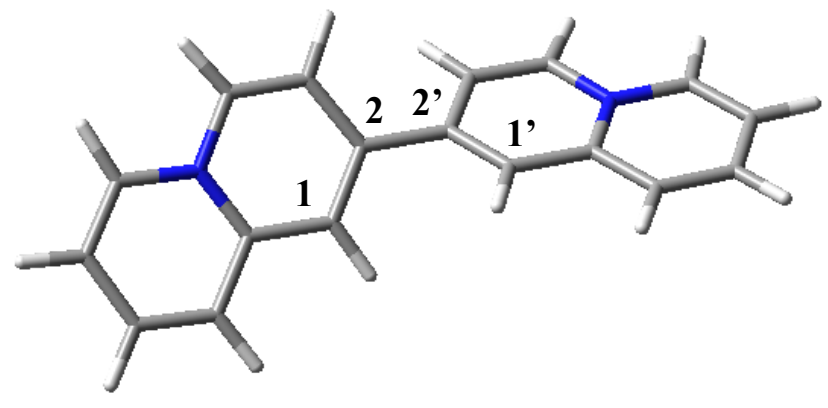

$\mathrm{E}_{\mathrm{HF} / 6-31 \mathrm{G}(\mathrm{d})}(\mathrm{a} . \mathrm{u})=-798.2033123$ ZPE (a.u.) $=0.298428$

Number of Imaginary Frequencies $=0$ 


\section{$\underline{\text { 2,2'-Biquinolizinium GS2 }}$}

$\begin{array}{rrrr}6 & -0.452729 & 0.925512 & 1.497055 \\ 6 & -0.409050 & 0.874433 & 2.917906 \\ 6 & -0.164500 & -0.163777 & 0.742707 \\ 1 & -0.700169 & 1.871149 & 1.054468 \\ 7 & -0.080589 & -0.299507 & 3.519154 \\ 6 & 0.175344 & -1.377459 & 1.416982 \\ 6 & -0.691287 & 1.996025 & 3.720447 \\ 6 & -0.191953 & -0.108270 & -0.746300 \\ 6 & 0.204957 & -1.416937 & 2.756115 \\ 6 & -0.639027 & 1.900770 & 5.076471 \\ 6 & -1.264485 & 0.546074 & -1.427973 \\ 6 & 0.792396 & -0.666153 & -1.493623 \\ 6 & -0.028977 & -0.396471 & 4.890978 \\ 1 & 0.384209 & -2.275914 & 0.870100 \\ 1 & -0.946044 & 2.917631 & 3.234445 \\ 6 & -0.298221 & 0.665987 & 5.674609 \\ 6 & 0.772708 & -0.596830 & -2.914242 \\ 6 & -1.277724 & 0.597994 & -2.766938 \\ 1 & 0.440919 & -2.303952 & 3.307205 \\ 1 & -0.854243 & 2.753792 & 5.691778 \\ 1 & -2.082995 & 0.979183 & -0.887247 \\ 1 & 1.633719 & -1.158378 & -1.045033 \\ 1 & 0.235955 & -1.358924 & 5.278079 \\ 7 & -0.265431 & 0.035320 & -3.522652 \\ 6 & 1.790408 & -1.156982 & -3.709355 \\ 1 & -0.249641 & 0.560351 & 6.740807 \\ 1 & -2.064102 & 1.064819 & -3.323587 \\ 6 & -0.327900 & 0.123962 & -4.894582 \\ 6 & 1.729548 & -1.068337 & -5.065473 \\ 1 & 2.606060 & -1.650374 & -3.217690 \\ 6 & 0.635941 & -0.408261 & -5.671207 \\ 1 & -1.181504 & 0.637018 & -5.287627 \\ 1 & 2.503482 & -1.494836 & -5.675139 \\ 1 & 0.560422 & -0.323209 & -6.737679\end{array}$



$\mathrm{E}_{\mathrm{HF} / 6-31 \mathrm{G}(\mathrm{d})}(\mathrm{a} . \mathrm{u})=-798.2031503$

ZPE (a.u.) $=0.29844$

Number of Imaginary Frequencies $=0$ 


$\begin{array}{rrrc}6 & -0.605353 & -0.244781 & 1.461447 \\ 6 & -0.711001 & -0.116432 & 2.872216 \\ 6 & 0.363555 & 0.387690 & 0.747645 \\ 1 & -1.340692 & -0.872839 & 1.003071 \\ 7 & 0.185476 & 0.663714 & 3.528877 \\ 6 & 1.282311 & 1.197520 & 1.490224 \\ 6 & -1.714226 & -0.771022 & 3.613135 \\ 6 & 0.479242 & 0.255729 & -0.739174 \\ 6 & 1.176025 & 1.313500 & 2.820764 \\ 6 & -1.778922 & -0.620434 & 4.963385 \\ 6 & 1.511885 & 0.935676 & -1.462141 \\ 6 & -0.377043 & -0.505226 & -1.471320 \\ 6 & 0.121801 & 0.817338 & 4.894379 \\ 1 & 2.078626 & 1.734641 & 1.020557 \\ 1 & -2.418052 & -1.385210 & 3.086143 \\ 6 & -0.831087 & 0.198682 & 5.618989 \\ 6 & -0.263161 & -0.627289 & -2.882006 \\ 6 & 1.612650 & 0.815481 & -2.792736 \\ 1 & 1.846266 & 1.910468 & 3.404315 \\ 1 & -2.542097 & -1.117723 & 5.531541 \\ 1 & 2.233825 & 1.557652 & -0.977215 \\ 1 & -1.182419 & -1.053411 & -1.028428 \\ 1 & 0.869685 & 1.450876 & 5.325342 \\ 7 & 0.733889 & 0.038160 & -3.519636 \\ 6 & -1.149479 & -1.415253 & -3.641916 \\ 1 & -0.857415 & 0.336032 & 6.682349 \\ 1 & 2.372474 & 1.310282 & -3.361915 \\ 6 & 0.882635 & -0.050517 & -4.884284 \\ 6 & -1.004124 & -1.504262 & -4.991355 \\ 1 & -1.934044 & -1.937362 & -3.129882 \\ 6 & 0.044033 & -0.799557 & -5.626898 \\ 1 & 1.696253 & 0.508059 & -5.299553 \\ 1 & -1.677660 & -2.103816 & -5.573950 \\ 1 & 0.183085 & -0.850849 & -6.689126\end{array}$

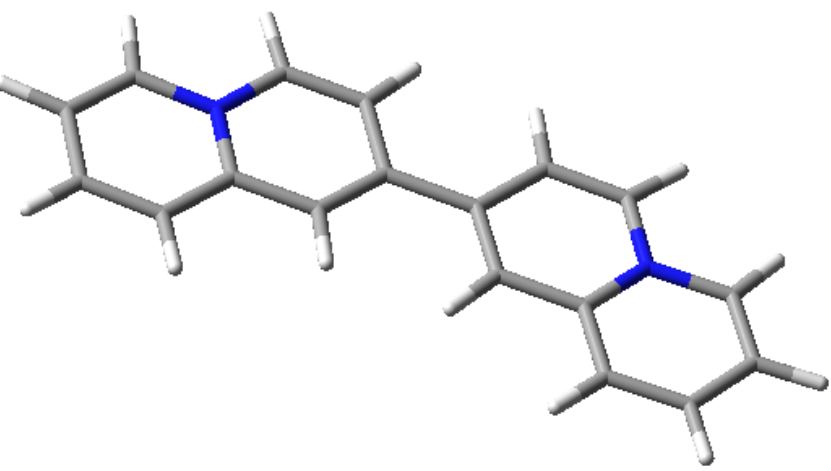

$\mathrm{E}_{\mathrm{HF} / 6-31 \mathrm{G}(\mathrm{d})}(\mathrm{a} . \mathrm{u})=-798.1980314$ $\mathrm{ZPE}$ (a.u.) $=0.298542$

Number of Imaginary Frequencies $=1$ $v\left(\mathrm{~cm}^{-1}\right)=-66.7922$ 


\section{2,2'-Biquinolizinium anti-TS}

$\begin{array}{rrrr}6 & 1.677121 & -0.818338 & 0.000046 \\ 6 & 3.069118 & -0.521555 & 0.000048 \\ 6 & 0.730814 & 0.155634 & -0.000041 \\ 1 & 1.433764 & -1.860031 & 0.000114 \\ 7 & 3.462165 & 0.777281 & -0.000048 \\ 6 & 1.201457 & 1.510508 & -0.000142 \\ 6 & 4.050596 & -1.529443 & 0.000144 \\ 6 & -0.730814 & -0.155621 & -0.000042 \\ 6 & 2.511631 & 1.781787 & -0.000143 \\ 6 & 5.371771 & -1.200786 & 0.000140 \\ 6 & -1.201450 & -1.510497 & -0.000134 \\ 6 & -1.677125 & 0.818347 & 0.000054 \\ 6 & 4.794822 & 1.111774 & -0.000052 \\ 1 & 0.532983 & 2.345359 & -0.000215 \\ 1 & 3.730858 & -2.553229 & 0.000219 \\ 6 & 5.750260 & 0.160179 & 0.000040 \\ 6 & -3.069121 & 0.521556 & 0.000052 \\ 6 & -2.511623 & -1.781783 & -0.000137 \\ 1 & 2.896299 & 2.780820 & -0.000219 \\ 1 & 6.123816 & -1.966959 & 0.000214 \\ 1 & -0.532971 & -2.345345 & -0.000191 \\ 1 & -1.433775 & 1.860041 & 0.000134 \\ 1 & 5.010604 & 2.160545 & -0.000131 \\ 7 & -3.462161 & -0.777282 & -0.000048 \\ 6 & -4.050605 & 1.529439 & 0.000149 \\ 1 & 6.783006 & 0.449497 & 0.000034 \\ 1 & -2.896286 & -2.780818 & -0.000205 \\ 6 & -4.794818 & -1.111781 & -0.000058 \\ 6 & -5.371777 & 1.200776 & 0.000140 \\ 1 & -3.730872 & 2.553226 & 0.000229 \\ 6 & -5.750260 & -0.160191 & 0.000033 \\ 1 & -5.010594 & -2.160554 & -0.000141 \\ 1 & -6.123826 & 1.966944 & 0.000214 \\ 1 & -6.783004 & -0.449515 & 0.000023\end{array}$

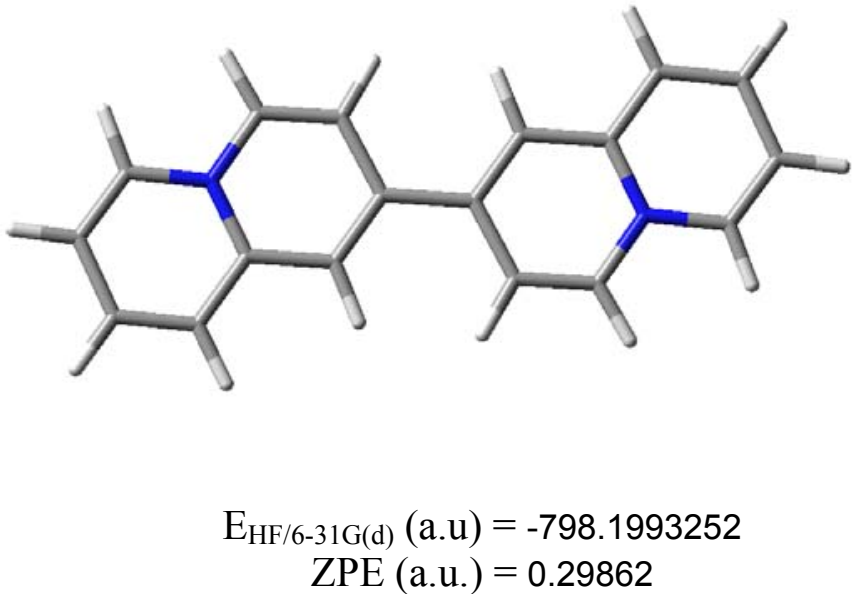

Number of Imaginary Frequencies $=1$ $v\left(\mathrm{~cm}^{-1}\right)=-58.1916$ 


\section{2,2'-Biquinolizinium GS1GS2-TS}

\begin{tabular}{|c|c|c|}
\hline-1.564220 & 0.443491 & 0.619815 \\
\hline-2.972426 & 0.428384 & 0.410084 \\
\hline-0.740989 & -0.345151 & -0.109217 \\
\hline-1.187974 & 1.105806 & 1.375788 \\
\hline-3.481330 & -0.398898 & -0.540725 \\
\hline-1.314922 & -1.200982 & -1.098110 \\
\hline-3.852601 & 1.238589 & 1.152031 \\
\hline 0.740989 & -0.345151 & 0.109217 \\
\hline-2.642511 & -1.206874 & -1.286518 \\
\hline-5.191712 & 1.190733 & 0.918480 \\
\hline 1.314922 & -1.200982 & 1.098110 \\
\hline 1.564220 & 0.443491 & -0.619815 \\
\hline-4.837041 & -0.447177 & -0.777212 \\
\hline-0.700017 & -1.843714 & -1.697774 \\
\hline-3.437929 & 1.886921 & 1.899160 \\
\hline-5.693763 & 0.320531 & -0.076529 \\
\hline 2.972426 & 0.428384 & -0.410084 \\
\hline 2.642511 & -1.206874 & 1.286518 \\
\hline-3.125266 & -1.827087 & -2.013511 \\
\hline-5.866187 & 1.806715 & 1.482598 \\
\hline 0.700017 & -1.843714 & 1.697774 \\
\hline 1.187974 & 1.105806 & -1.375788 \\
\hline-5.149776 & -1.126632 & -1.543279 \\
\hline 3.481330 & -0.398898 & 0.540725 \\
\hline 3.852601 & 1.238589 & -1.152031 \\
\hline-6.744544 & 0.261022 & -0.283001 \\
\hline 3.125266 & -1.827087 & 2.013511 \\
\hline 4.837041 & -0.447177 & 0.777212 \\
\hline 5.191712 & 1.190733 & -0.918481 \\
\hline 3.437929 & 1.886920 & -1.899160 \\
\hline 5.693763 & 0.320531 & 0.076529 \\
\hline 5.149776 & -1.126632 & 1.543279 \\
\hline 5.866187 & 1.806715 & -1.482598 \\
\hline 6.744544 & 0.261022 & 0.283001 \\
\hline
\end{tabular}

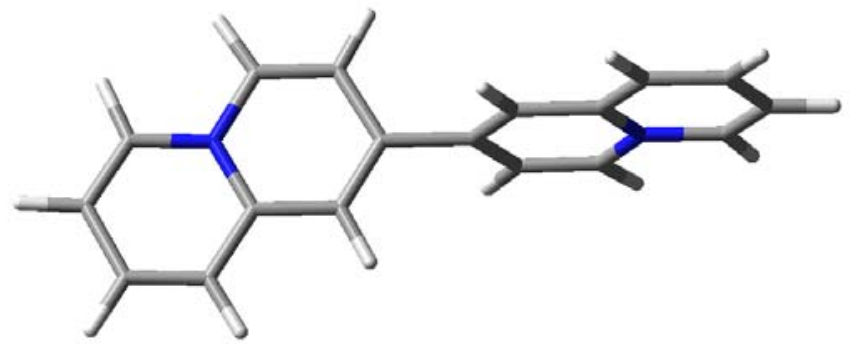

$\mathrm{E}_{\mathrm{HF} / 6-31 \mathrm{G}(\mathrm{d})}(\mathrm{a} . \mathrm{u})=-798.2014261$

$\mathrm{ZPE}$ (a.u.) $=0.298198$

Number of Imaginary Frequencies $=1$ $v\left(\mathrm{~cm}^{-1}\right)=-33.9227$ 


\section{$\underline{\text { 3,3'-Biquinolizinium GS1 }}$}

$\begin{array}{rrr}-4.392498 & 0.495799 & 2.266200 \\ -4.355381 & 0.193641 & 3.591154 \\ -3.213029 & 0.471426 & 1.497760 \\ -5.309369 & 0.759637 & 1.776028 \\ -3.119877 & -0.145576 & 4.191816 \\ -2.039051 & 0.137557 & 2.097468 \\ -3.197103 & 0.786960 & 0.110915 \\ -5.252126 & 0.212222 & 4.181122 \\ -2.002261 & -0.165680 & 3.441734 \\ -2.046516 & 0.755831 & -0.590711 \\ -0.859443 & 0.104907 & 1.374441 \\ -3.058328 & -0.386156 & 5.235230 \\ -4.128677 & 1.045893 & -0.353640 \\ -0.824408 & 0.394708 & 0.060780 \\ -1.037880 & -0.411869 & 3.835972 \\ -2.045228 & 0.988560 & -1.639257 \\ 0.012687 & -0.146409 & 1.942570 \\ 0.458506 & 0.367216 & -0.702899 \\ 0.939690 & 1.548757 & -1.351631 \\ 1.176511 & -0.765089 & -0.818048 \\ 2.094009 & 1.509785 & -2.046701 \\ 2.361160 & -0.808224 & -1.532232 \\ 0.386204 & 2.465784 & -1.272087 \\ 0.873065 & -1.697683 & -0.388250 \\ 2.840737 & 0.303694 & -2.151089 \\ 3.043875 & -2.003045 & -1.613880 \\ 2.482266 & 2.382097 & -2.535594 \\ 4.048350 & 0.220802 & -2.870148 \\ 4.198768 & -2.100744 & -2.298548 \\ 2.594985 & -2.830140 & -1.103515 \\ 4.722216 & -0.957382 & -2.947434 \\ 4.412319 & 1.108251 & -3.350242 \\ 4.706122 & -3.044639 & -2.343628 \\ 5.642741 & -1.025370 & -3.495676\end{array}$

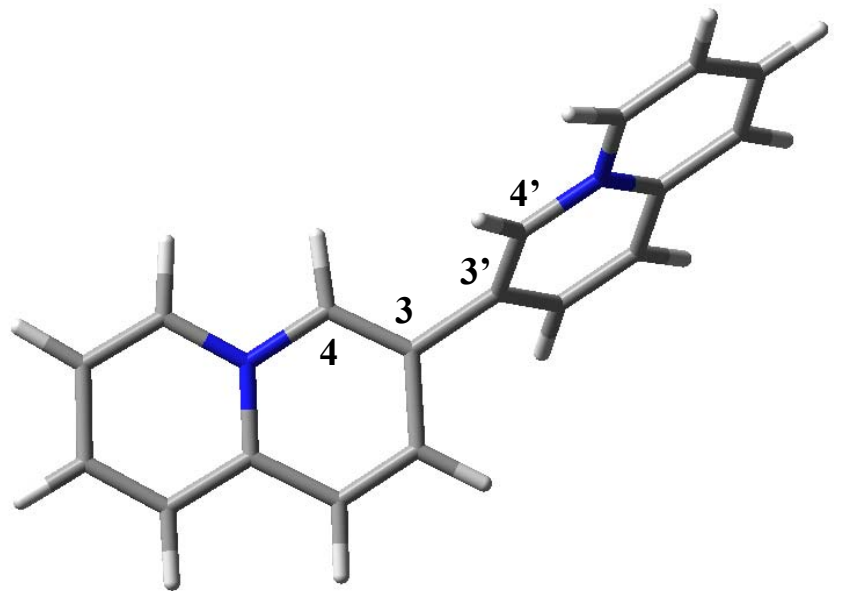

$\mathrm{E}_{\mathrm{HF} / 6-31 \mathrm{G}(\mathrm{d})}(\mathrm{a} . \mathrm{u})=-798.1984647$

ZPE (a.u.) $=0.298337$

Number of Imaginary Frequencies $=0$ 


\section{3,3'-Biquinolizinium GS2}

$\begin{array}{rrr}-4.430543 & 0.090011 & 2.209192 \\ -4.459428 & 0.424860 & 3.526075 \\ -3.215666 & 0.107581 & 1.496988 \\ -5.320752 & -0.193424 & 1.682192 \\ -3.256727 & 0.791442 & 4.176175 \\ -2.074040 & 0.466436 & 2.143712 \\ -3.130731 & -0.232903 & 0.119854 \\ -5.383153 & 0.412643 & 4.072976 \\ -2.104593 & 0.804124 & 3.480683 \\ -1.948207 & -0.200183 & -0.528249 \\ -0.863712 & 0.496059 & 1.477444 \\ -3.247650 & 1.059934 & 5.214553 \\ -4.037349 & -0.504334 & -0.385159 \\ -0.760341 & 0.176630 & 0.172764 \\ -1.162708 & 1.071464 & 3.913622 \\ -1.899094 & -0.437605 & -1.574263 \\ -0.024279 & 0.778319 & 2.079813 \\ 0.563443 & 0.231837 & -0.517639 \\ 1.360869 & 1.418534 & -0.500500 \\ 1.029808 & -0.840550 & -1.186519 \\ 2.548294 & 1.441084 & -1.140018 \\ 2.244462 & -0.819903 & -1.845212 \\ 1.003403 & 2.295568 & 0.005423 \\ 0.503142 & -1.771579 & -1.241066 \\ 3.020515 & 0.297254 & -1.838804 \\ 2.659871 & -1.955898 & -2.508092 \\ 3.161468 & 2.321201 & -1.148986 \\ 4.251880 & 0.280617 & -2.522128 \\ 3.834068 & -1.988986 & -3.164939 \\ 1.991720 & -2.791042 & -2.461572 \\ 4.659021 & -0.838846 & -3.176482 \\ 4.849314 & 1.171352 & -2.507938 \\ 4.128651 & -2.887358 & -3.671445 \\ 5.596968 & -0.856134 & -3.698472\end{array}$

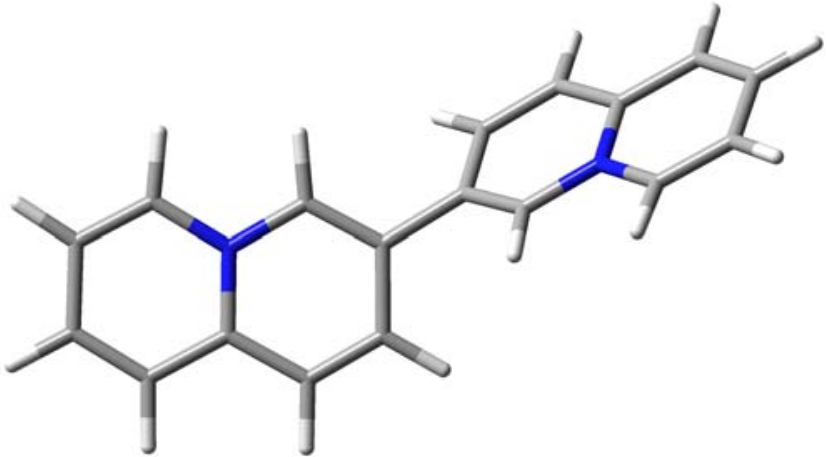

$\mathrm{E}_{\mathrm{HF} / 6-31 \mathrm{G}(\mathrm{d})}(\mathrm{a} . \mathrm{u})=-798.1980341$

ZPE (a.u.) $=0.29831$

Number of Imaginary Frequencies $=0$ 


$\begin{array}{rrc}-4.576635 & -0.003271 & 2.017907 \\ -4.525462 & -0.001187 & 3.375416 \\ -3.388021 & -0.002761 & 1.262225 \\ -5.510303 & -0.005336 & 1.489919 \\ -3.265297 & 0.001500 & 4.021558 \\ -2.190609 & -0.000164 & 1.903588 \\ -3.377073 & -0.004850 & -0.155706 \\ -5.427490 & -0.001562 & 3.957421 \\ -2.140652 & 0.001950 & 3.283413 \\ -2.216651 & -0.004277 & -0.841392 \\ -1.005004 & 0.000413 & 1.195702 \\ -3.191903 & 0.003187 & 5.091603 \\ -4.320786 & -0.006901 & -0.665399 \\ -0.958753 & -0.001531 & -0.154422 \\ -1.160687 & 0.003934 & 3.713712 \\ -2.262601 & -0.005925 & -1.910215 \\ -0.139782 & 0.002536 & 1.822086 \\ 0.337628 & -0.000875 & -0.910528 \\ 0.359010 & -0.002974 & -2.343630 \\ 1.535546 & 0.001695 & -0.286058 \\ 1.527158 & -0.002368 & -3.016068 \\ 2.735416 & 0.002328 & -0.969486 \\ -0.548670 & -0.005065 & -2.909855 \\ 1.654763 & 0.003433 & 0.775429 \\ 2.766714 & 0.000358 & -2.327486 \\ 3.911819 & 0.005014 & -0.246653 \\ 1.548154 & -0.003932 & -4.088421 \\ 4.009668 & 0.001085 & -2.990001 \\ 5.108013 & 0.005745 & -0.862122 \\ 3.803869 & 0.006442 & 0.818165 \\ 5.166048 & 0.003728 & -2.277096 \\ 4.009794 & -0.000505 & -4.062618 \\ 6.003244 & 0.007849 & -0.271409 \\ 6.116734 & 0.004296 & -2.775675\end{array}$

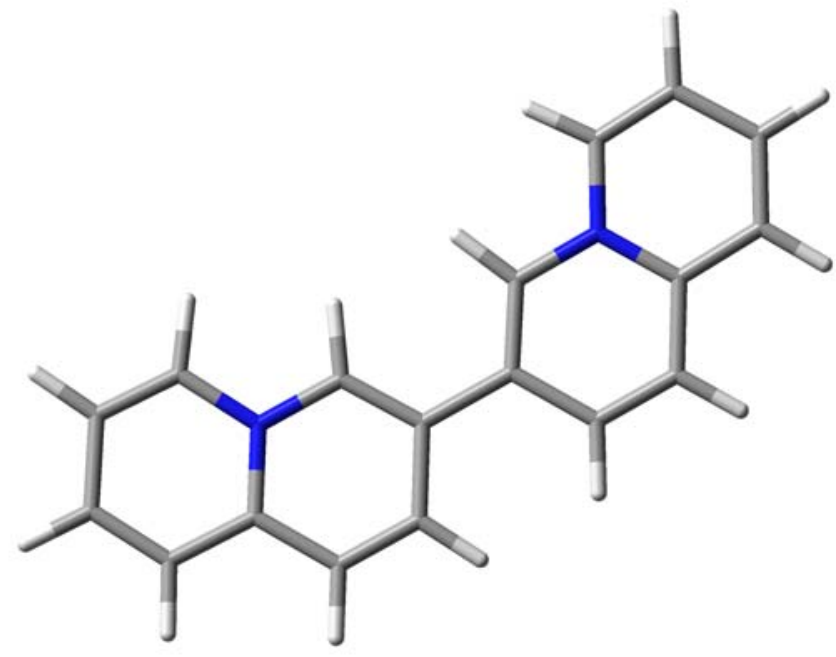

$\mathrm{E}_{\mathrm{HF} / 6-31 \mathrm{G}(\mathrm{d})}(\mathrm{a} . \mathrm{u})=-798.1899171$

ZPE (a.u.) $=0.298457$

Number of Imaginary Frequencies $=1$ $v\left(\mathrm{~cm}^{-1}\right)=-78.0310$ 


\section{3,3'-Biquinolizinium anti-TS}

$\begin{array}{rrc}-4.266574 & 0.002681 & 2.540129 \\ -4.213350 & 0.004088 & 3.899269 \\ -3.080205 & 0.001884 & 1.784918 \\ -5.201442 & 0.002147 & 2.014291 \\ -2.954235 & 0.004741 & 4.543721 \\ -1.882921 & 0.002534 & 2.424956 \\ -3.070368 & 0.000408 & 0.363792 \\ -5.115083 & 0.004703 & 4.481811 \\ -1.829982 & 0.003961 & 3.802747 \\ -1.912166 & -0.000315 & -0.321605 \\ -0.695531 & 0.001787 & 1.714957 \\ -2.879071 & 0.005849 & 5.613619 \\ -4.015381 & -0.000110 & -0.143500 \\ -0.652541 & 0.000387 & 0.366223 \\ -0.849211 & 0.004394 & 4.231487 \\ -1.958854 & -0.001428 & -1.391302 \\ 0.170223 & 0.002425 & 2.339030 \\ 0.652542 & -0.000382 & -0.366223 \\ 1.912166 & 0.000319 & 0.321605 \\ 0.695531 & -0.001779 & -1.714957 \\ 3.070368 & -0.000402 & -0.363792 \\ 1.882922 & -0.002527 & -2.424956 \\ 1.958854 & 0.001430 & 1.391302 \\ -0.170222 & -0.002417 & -2.339030 \\ 3.080205 & -0.001875 & -1.784918 \\ 1.829982 & -0.003950 & -3.802748 \\ 4.015381 & 0.000116 & 0.143500 \\ 4.266575 & -0.002670 & -2.540129 \\ 2.954235 & -0.004728 & -4.543721 \\ 0.849211 & -0.004384 & -4.231488 \\ 4.213351 & -0.004075 & -3.899270 \\ 5.201442 & -0.002136 & -2.014291 \\ 2.879072 & -0.005833 & -5.613619 \\ 5.115084 & -0.004688 & -4.481811\end{array}$

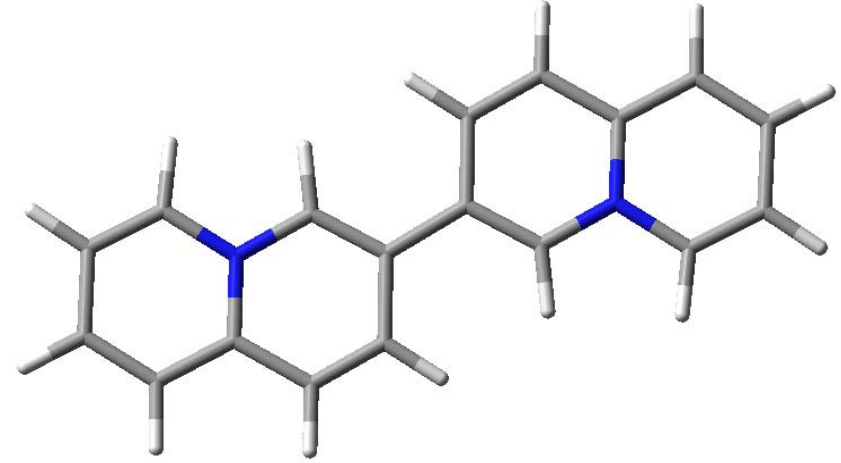

$\mathrm{E}_{\mathrm{HF} / 6-31 \mathrm{G}(\mathrm{d})}(\mathrm{a} . \mathrm{u})=-798.1926146$

ZPE (a.u.) $=0.298545$

Number of Imaginary Frequencies $=1$ $v\left(\mathrm{~cm}^{-1}\right)=-64.4634$ 


\section{3,3'-Biquinolizinium GS1GS2-TS}

$\begin{array}{rrr}-4.433591 & 0.264607 & 2.206427 \\ -4.413741 & 0.324404 & 3.563932 \\ -3.228865 & 0.261838 & 1.476309 \\ -5.355329 & 0.219295 & 1.659736 \\ -3.170929 & 0.383371 & 4.239144 \\ -2.047866 & 0.319326 & 2.148891 \\ -3.195334 & 0.202567 & 0.056653 \\ -5.329549 & 0.327418 & 4.124146 \\ -2.029250 & 0.379473 & 3.526698 \\ -2.020390 & 0.205031 & -0.606873 \\ -0.845212 & 0.319554 & 1.466120 \\ -3.123558 & 0.431728 & 5.309566 \\ -4.131937 & 0.159777 & -0.464553 \\ -0.792387 & 0.263456 & 0.122861 \\ -1.058589 & 0.422821 & 3.976200 \\ -2.001863 & 0.166738 & -1.679881 \\ 0.027662 & 0.367289 & 2.084979 \\ 0.524737 & 0.293551 & -0.592364 \\ 1.184737 & 1.533411 & -0.858933 \\ 1.097995 & -0.845053 & -1.022757 \\ 2.358256 & 1.538828 & -1.524960 \\ 2.299555 & -0.842564 & -1.707445 \\ 0.741622 & 2.454851 & -0.530674 \\ 0.667603 & -1.813145 & -0.865480 \\ 2.947135 & 0.324427 & -1.970506 \\ 2.830796 & -2.046415 & -2.120777 \\ 2.875528 & 2.453818 & -1.739280 \\ 4.168558 & 0.286917 & -2.671337 \\ 3.996240 & -2.099757 & -2.791536 \\ 2.257360 & -2.915985 & -1.873525 \\ 4.690556 & -0.899925 & -3.078415 \\ 4.665113 & 1.216604 & -2.870438 \\ 4.383080 & -3.051276 & -3.100330 \\ 5.620774 & -0.932923 & -3.613352 \\ & & \end{array}$

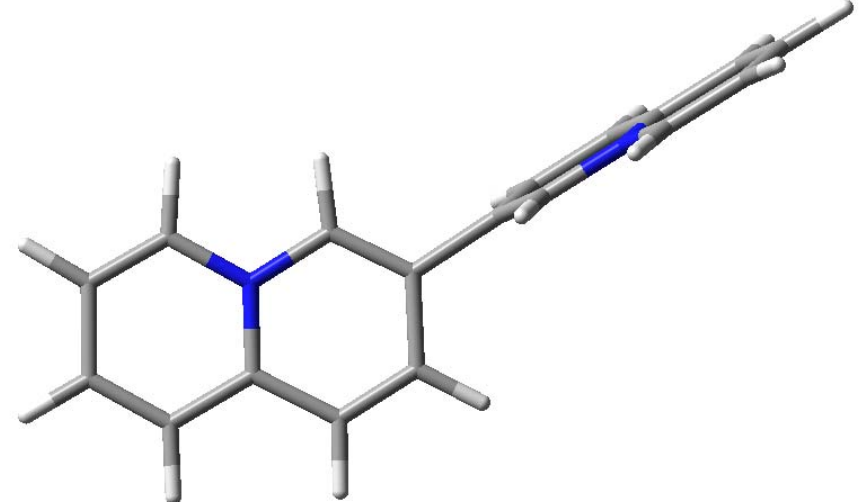

$\mathrm{E}_{\mathrm{HF} / 6-31 \mathrm{G}(\mathrm{d})}(\mathrm{a} . \mathrm{u})=-798.1976455$

ZPE (a.u.) $=0.298196$

Number of Imaginary Frequencies $=1$ $v\left(\mathrm{~cm}^{-1}\right)=-23.3449$ 


\section{$\underline{\text { 4,4'-Biquinolizinium GS }}$}

$\begin{array}{rrr}-3.357973 & -0.715058 & -1.215531 \\ -3.369073 & -0.762617 & 0.130580 \\ -2.130045 & -0.626232 & -1.928645 \\ -4.263750 & -0.749608 & -1.789299 \\ -2.129317 & -0.711910 & 0.827621 \\ -0.953321 & -0.579367 & -1.239173 \\ -2.110039 & -0.592252 & -3.337809 \\ -4.288667 & -0.837639 & 0.678928 \\ -0.961898 & -0.617268 & 0.164684 \\ -0.935229 & -0.520039 & -4.014660 \\ 0.236593 & -0.514403 & -1.934496 \\ -3.051113 & -0.630173 & -3.851178 \\ -2.115871 & -0.748189 & 1.900107 \\ 0.271709 & -0.482148 & -3.280746 \\ -0.918300 & -0.496987 & -5.087805 \\ 1.127214 & -0.505932 & -1.346175 \\ 0.332935 & -0.620610 & 0.915088 \\ 1.221227 & -0.434795 & -3.777667 \\ 0.961954 & 0.596360 & 1.224351 \\ 0.863130 & -1.780104 & 1.346104 \\ 0.414298 & 1.795439 & 0.817079 \\ 2.116863 & 0.600287 & 1.951275 \\ 2.066341 & -1.801325 & 2.105885 \\ 0.364017 & -2.699683 & 1.107389 \\ 2.737733 & 1.827632 & 2.260217 \\ 0.995433 & 2.976044 & 1.105380 \\ 2.672992 & -0.633073 & 2.391472 \\ -0.502588 & 1.735274 & 0.273849 \\ 2.472270 & -2.737261 & 2.439371 \\ 2.196855 & 3.003080 & 1.848763 \\ 3.644441 & 1.797917 & 2.832582 \\ 0.530728 & 3.882865 & 0.769998 \\ 3.581643 & -0.592866 & 2.960306 \\ 2.666995 & 3.938640 & 2.085734\end{array}$

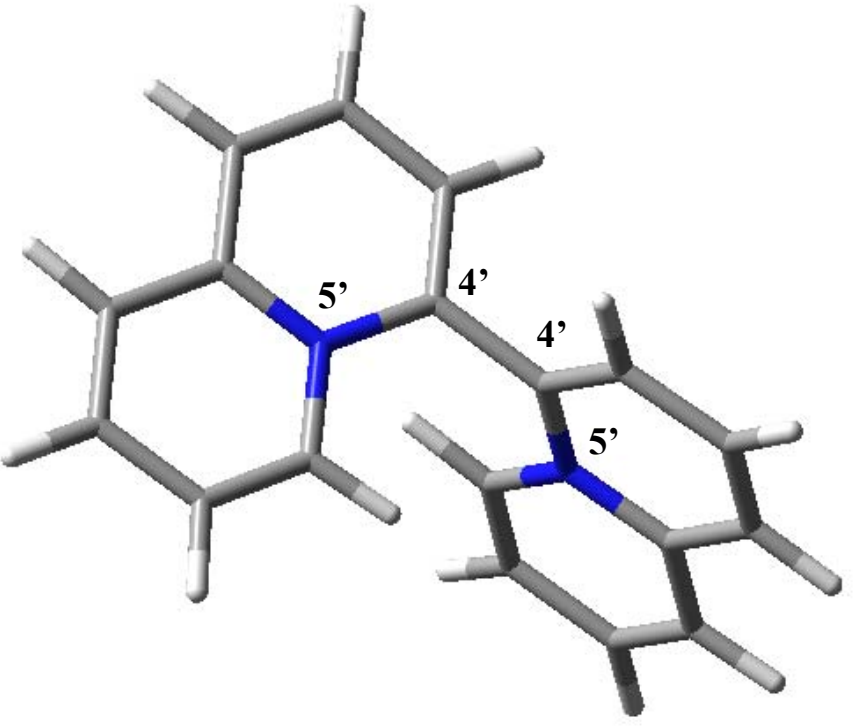

$\mathrm{E}_{\mathrm{HF} / 6-31 \mathrm{G}(\mathrm{d})}(\mathrm{a} . \mathrm{u})=-798.1723628$ $\mathrm{ZPE}$ (a.u.) $=0.298081$

Number of Imaginary Frequencies $=0$ 


\section{4,4'-Biquinolizinium anti-TS}

$\begin{array}{rrr}-3.140949 & -0.095381 & -1.169513 \\ -2.785042 & 1.137389 & -0.782914 \\ -2.130640 & -0.983062 & -1.636129 \\ -4.164141 & -0.408394 & -1.241132 \\ -1.404970 & 1.418286 & -0.556745 \\ -0.818970 & -0.600277 & -1.599190 \\ -2.495012 & -2.191207 & -2.255588 \\ -3.514907 & 1.887975 & -0.547329 \\ -0.416013 & 0.527577 & -0.800387 \\ -1.579112 & -2.931742 & -2.936418 \\ 0.046468 & -1.260360 & -2.438958 \\ -3.526244 & -2.482452 & -2.206612 \\ -1.210609 & 2.355840 & -0.103603 \\ -0.283596 & -2.401931 & -3.080977 \\ -1.854199 & -3.852676 & -3.414586 \\ 0.962774 & -0.773886 & -2.653414 \\ 0.927083 & 0.476332 & -0.075194 \\ 0.436263 & -2.858874 & -3.732042 \\ 1.009298 & 0.974143 & 1.273116 \\ 2.004129 & -0.241342 & -0.469753 \\ 0.402417 & 2.142107 & 1.669924 \\ 1.807656 & 0.348618 & 2.189474 \\ 2.987890 & -0.738926 & 0.435386 \\ 2.133024 & -0.543916 & -1.476708 \\ 1.741865 & 0.711786 & 3.545899 \\ 0.354601 & 2.541398 & 2.959341 \\ 2.801017 & -0.572352 & 1.751889 \\ 0.060764 & 2.784423 & 0.899879 \\ 3.805241 & -1.317663 & 0.049919 \\ 0.987039 & 1.768719 & 3.950777 \\ 2.340121 & 0.152529 & 4.238708 \\ -0.124624 & 3.471018 & 3.198151 \\ 3.435343 & -1.023196 & 2.489723 \\ 0.937534 & 2.052051 & 4.985045\end{array}$



$\mathrm{E}_{\mathrm{HF} / 6-31 \mathrm{G}(\mathrm{d})}(\mathrm{a} . \mathrm{u})=-798.1015063$

$\mathrm{ZPE}$ (a.u.) $=0.297800$

Number of Imaginary Frequencies $=1$ $v\left(\mathrm{~cm}^{-1}\right)=-101.4917$ 